\title{
2-Arylhydrazononitriles as building blocks in heterocyclic synthesis: A novel route to 2-substituted-1,2,3-triazoles and 1,2,3-triazolo[4,5-b] pyridines
}

Saleh M. Al-Mousawi ${ }^{*}$ and Moustafa Sh. Moustafa

\section{Full Research Paper}

Address:

Department of Chemistry, Faculty of Science; University of Kuwait:

Safat; 13060: Kuwait, P.O. Box 12613

Email:

Saleh M. Al-Mousawi* - salehalmousawi@hotmail.com;

Moustafa Sh. Moustafa - mostafa_msm@hotmail.com

* Corresponding author
Open Access

Beilstein Journal of Organic Chemistry 2007, 3, No. 12. doi:10.1186/1860-5397-3-12

Received: 11 November 2006

Accepted: 13 March 2007

Published: 13 March 2007

C 2007 Al-Mousawi and Moustafa; licensee Beilstein-Institut

License and terms: see end of document.

\begin{abstract}
2-Arylhydrazono-3-oxobutanenitriles 2 was reacted with hydroxylamine hydrochloride to yield amidooxime $\mathbf{3}$. This was cyclized into the corresponding oxadiazole 4 on refluxing in acetic anhydride. When refluxed in DMF in presence of piperidine, the corresponding 1,2,3-triazoleamine 5 was formed. The latter was acylated to $\mathbf{6}$ by addition of acetic anhydride while treatment of $\mathbf{5}$ with malononitrile gave the 1,2,3-triazolo [4,5-b]pyridine 8. Treatment of acetyl derivative 6 with DMFDMA gave enaminone 9. The enaminone 9 was coupled with benzenediazonium chloride to yield phenylazo-1,2,3-triazolo [4,5-b]pyridine 10. Trials to convert compound 14 into 1,2,3-triazolo [4,5- $d$ ] pyrimidine 15 via refluxing in $\mathrm{AcOH} / \mathrm{NH}_{4} \mathrm{OAc}$ failed. Instead the hydrolyzed product 5 was formed.
\end{abstract}

\section{Introduction}

Interest in 1,2,3-triazole chemistry has recently been revived. [1-4] Zaprinast (1) is used as cyclic AMP phosphodiasterase inhibitor. [5] Plenty of 1,2,3-triazoles have also been reported to have useful pharmaceutical potential. [6-9] Synthetic approaches to 1,2,3-triazoloazines are rather limited. There are in fact few known routes to 4-functionally substituted 1,2,3triazole-5-amines. [7] The latter is one of the logical starting points for condensed 1,2,3-triazoloazines. In the present article we provide a versatile route to these derivatives.

\section{Results and discussion}

Elnagdi et al [10] have reported that arylhydrazononitriles reacted with hydroxylamine hydrochloride in ethanolic sodium acetate yielded amidooxime 3 that cyclized into 

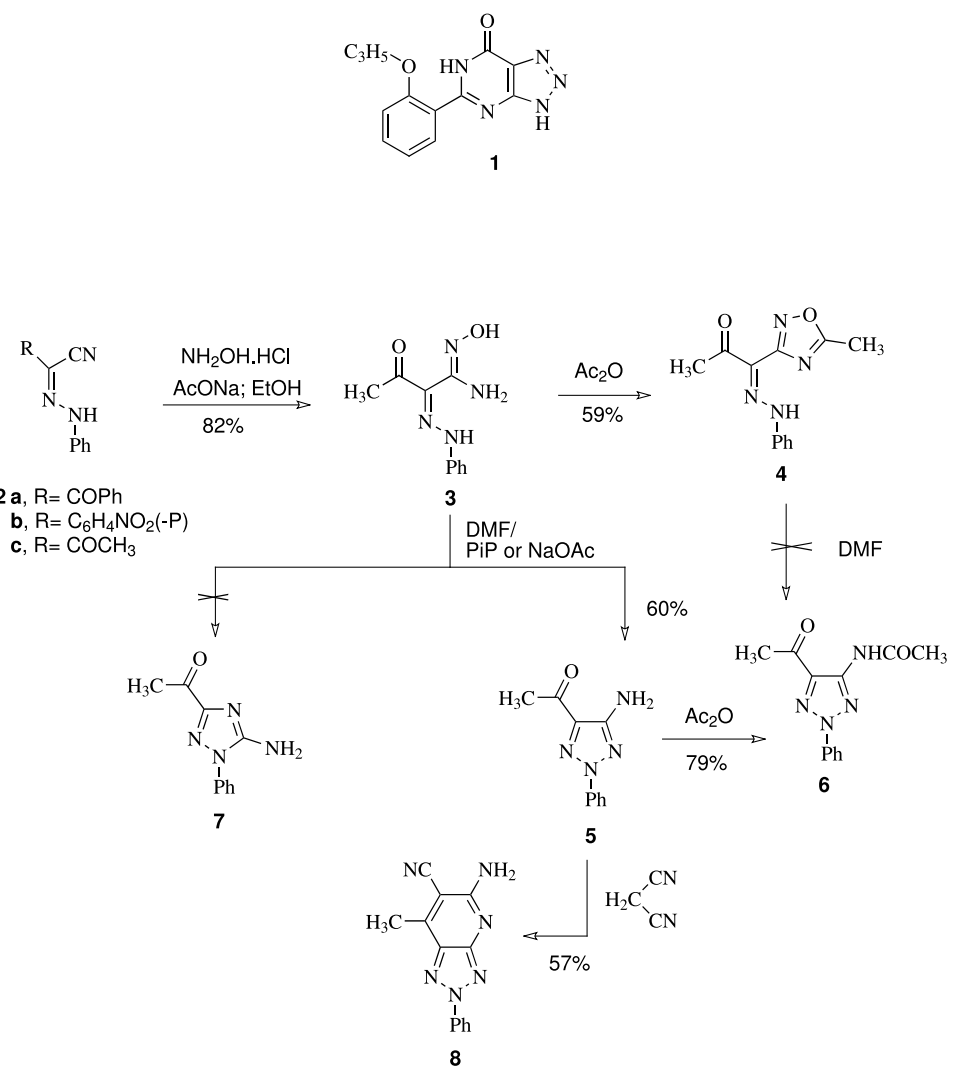

Scheme 1: Synthesis of triazolopyridine 8.

aminoisoxazoles on treatment with mineral acids. Moreover, recently it was shown that refluxing $\mathbf{2 a}$ with hydroxylamine hydrochloride in DMF/piperidine afforded 1,2,3-triazoles whose structure was established by X-ray crystal structure. [11] On the other hand reacting $\mathbf{2 b}$ with hydroxylamine hydrochloride in DMF/piperidine afforded 5-amino-1,2,4-triazole via a rare Tiemann-like rearrangement. In order to clarify the rule of the substituent and the reaction condition we have investigated the behavior of compound $\mathbf{2 c}$ with hydroxylamine hydrochloride in DMF/piperidine.

Reacting 2c with hydroxylamine hydrochloride in ethanolic sodium acetate afforded the corresponding amidooxime $\mathbf{3}$ in good yield. Formation of amidooximes on reacting 2-arylhydrazononitriles with hydroxylamine hydrochloride under similar conditions has been reported earlier by Elnagdi et al. [10,11] Attempted cyclization of 3 into 1,2,3-triazole via reflux in acetic anhydride, as has been reported recently, [11] afforded a product that can be assigned as 1,2,4-oxadiazolylphenylhydrazone structure 4 or the isomeric acetylamino-1,2,3-triazole structure 6. Phenylhydrazono-1,2,4-oxadiazoles have been reported to rearrange thermally into acylamino-1,2,3-triazoles. [12] Compound 5 could be obtained via refluxing 3 in DMF in the presence of piperidine or anhydrous sodium acetate. Acylation of the latter afforded the acetylamino-1,2,3-triazole 6 which proved to be different in all respects from 4 . To exclude the possible rearrangement of $\mathbf{3}$ prior to cyclization (Tiemann rearrangement), [13] as has been noted recently by Al-Matar et $a l,[14]$ and the possibility that the really formed product is the 5-amino-1,2,4-triazole 7. We decided to investigate further the chemical reactivity pattern of the cyclized product (Scheme 1) to support our conclusion.

Compound 5 was reacted with malononitrile in refluxing DMF to yield the triazolopyridine $\mathbf{8}$, thus confirming that the aminofunction and the acetyl function are adjacent. Moreover, reacting 6 with dimethylformamide dimethylacetal (DMFDMA) afforded the cis enaminone $9(J=8 \mathrm{~Hz})$ despite the fact that secondary enaminones have been established to prefer adopting the trans configuration. [15] Compound 9 was coupled with benzenediazonium chloride most likely through the intermediate 11a. The latter intermediate cyclized into $\mathbf{1 1 b}$. Deacetylation of $11 \mathbf{b}$ followed by hydrogen shift produced 10. This again confirms that the acetyl and the amino functions in cyclization product are adjacent as in $\mathbf{5}$ and therefore excluding $\mathbf{7}$ (Scheme 2). Typical of enaminones, compound 9 reacts with naph- 


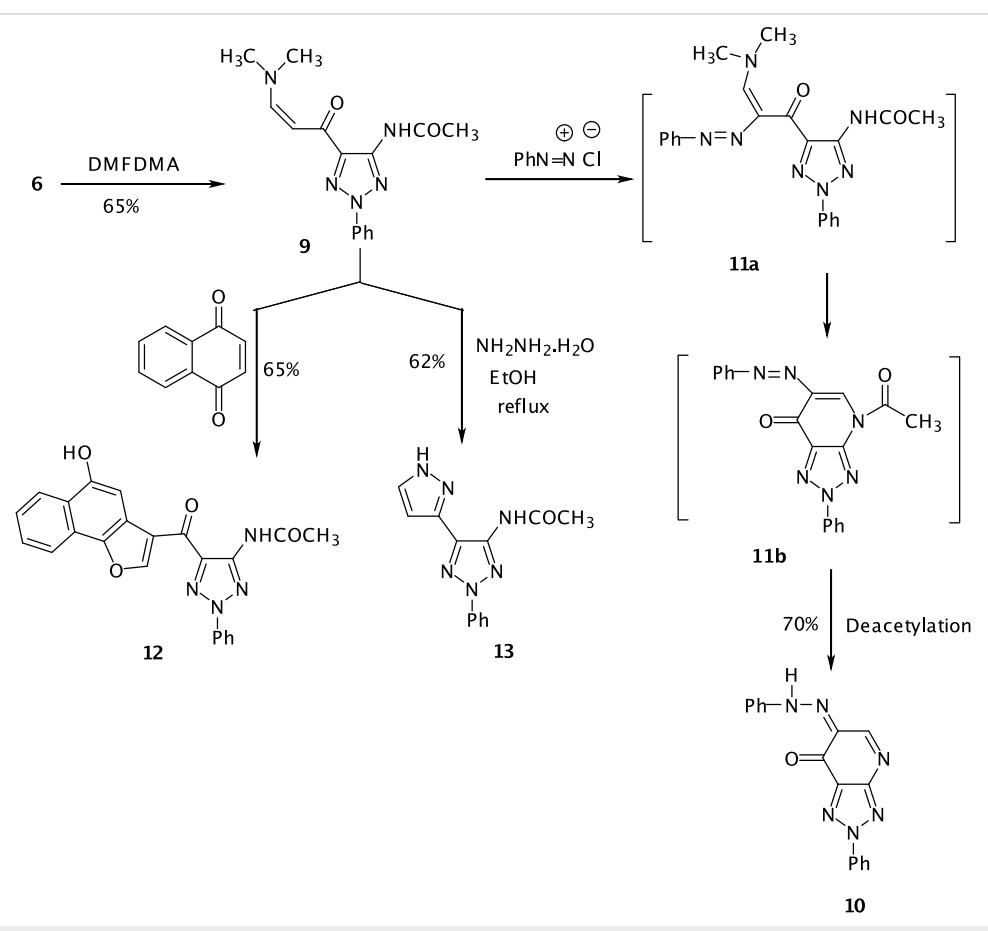

Scheme 2: Synthesis of 1,2,3-triazoles and 1,2,3-triazolo[4,5-b]pyridines.

thoquinone to yield the benzofuran 12 and with hydrazine hydrate to yield pyrazolyl-1,2,3-triazole $\mathbf{1 3}$ in good yields.

Attempts to convert 4 into oxadiazolylhydrazone $\mathbf{6}$ by heating in DMF afforded $\mathbf{1 4}$ whose structure could be elucidated through synthesis independently from $\mathbf{3}$. Trials to cyclise $\mathbf{1 4}$ into triazolopyrimidine $\mathbf{1 5}$ failed under a variety of conditions. It is thought that compound $\mathbf{1 5}$, if formed, has hydrolyzed to $\mathbf{5}$ (Scheme 3).

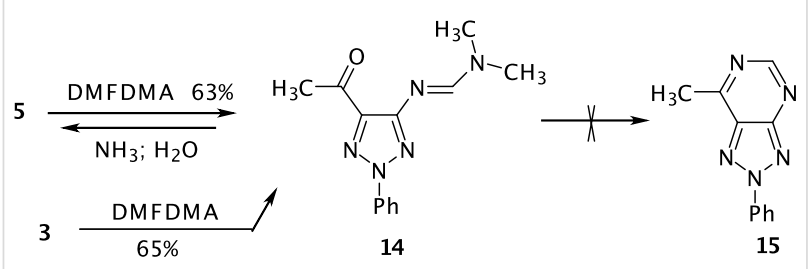

Scheme 3: Identification of 1,2,3-triazole 14.

\section{Conclusion}

An excellent simple route to 2-substituted-1,2,3-triazols has been reached. It could be also revealed that the formed triazoles are excellent precursors to condensed triazoles and azolyltriazoles.

\section{Experimental Section}

[See Supporting Information File 1]

\section{Supporting Information}

\section{Supporting Information File 1}

Experimental Section. the experimental data and the results of analysis

[http://www.beilstein-journals.org/bjoc/content/ supplementary/1860-5397-3-12-S1.doc]

\section{Acknowledgments}

The authors are grateful to Professor M. H. Elnagdi from Cairo University for interest and discussion. Also the authors are grateful to Kuwait University, Research Administration for financial support through project No. SC11/02 and for SAF facilities project Gs01/02; Gs03/01.

\section{References}

1. Invidiata, F. P.; Aiello, S.; Furno, G.; Aiello, E.; Simoni, D.; Rondanin, R. J. Heterocycl. Chem. 2000, 37, 355-361.

2. Lalezari, I.; Gomez, L. A.; Khorshidi, M. J. Heterocycl. Chem. 1990, 27 , 687-689.

3. Zhao, Y. B.; Yan, Z. Y.; Liang, Y. M. Tetrahedron Lett. 2006, 47, 1545-1549. doi:10.1016/j.tetlet.2006.01.004

4. Kiselyou, A. S. Tetrahedron Lett. 2006, 47, 2631-2634. doi:10.1016/ j.tetlet.2006.02.030

5. Taniguchi, Y.; Tonai-Kuchi, H.; Shinjo, K. FEBS Lett. 2006, 580, 5003-5008. doi:10.1016/j.febslet.2006.08.015

6. Kadaba, P. K. J. Med. Chem. 1988, 31, 196-203. doi:10.1021/ jm00396a032 
7. Wamhoff, H. In Comprehensive Heterocyclic Chemistry; Potts, K. T., Ed.; Pergamon press: Oxford, 1984; Vol. 5, pp 669 ff.

8. Ferrarini, P. L.; Livi, O. Farmaco, Ed. Sci. 1981, 83, 243.

9. Matloubi, H.; Shafiee, A.; Saemian, N.; Shirvani, G.; Daha, F. J. Appl. Radiat. Isot. 2004, 60, 665-668. doi:10.1016/j.apradiso.2003.12.003

10. Elnagdi, M. H.; Elmoghayar, M. R. H.; Hafez, E. A. A.; Alnima, H. H. J. Org. Chem. 1975, 40, 2604-2607. doi:10.1021/jo00906a007

11. Ghozlan, S. A. S.; Abdelhamid, I. A.; Elnagdi, M. H. ARKIVOC 2006, No. xv, 53-60.

12. Fan, W. Q.; Katritzky, A. R. Comprehensive Heterocyclic Chemistry II; Elsevier: New York, 1996; Vol. 4.

13. Tiemann, F. Ber. Dtsch. Chem. Ges. 1891, 24, 4162-4167.

14. Al-Matar, H.; Riyadh, S. M.; Elnagdi, M. H. Tetrahedron 2006, in press.

15. Al-Saleh, B.; El-Apasery, M. A.; Abdel-Aziz, R. S.; Elnagdi, M. H. J. Heterocycl. Chem. 2005, 42, 563-566.

\section{License and Terms}

This is an Open Access article under the terms of the Creative Commons Attribution License

(http://creativecommons.org/licenses/by/2.0), which permits unrestricted use, distribution, and reproduction in any medium, provided the original work is properly cited.

The license is subject to the Beilstein Journal of Organic Chemistry terms and conditions:

(http://www.beilstein-journals.org/bjoc)

The definitive version of this article is the electronic one which can be found at: doi:10.1186/1860-5397-3-12 